\title{
Centrifugal pump dynamic simulation experience for waterhammer calculation
}

\author{
Leonid Korelstein ${ }^{1 *}$, Vladimir Tarasevich ${ }^{2}$, and Elena Yudovina ${ }^{1}$ \\ ${ }^{1}$ Piping System Research \& Engineering Co (PSRE Co), Moscow, Russia \\ ${ }^{2}$ Novosibirsk State University of Architecture and Civil Engineering (Sibstrin), Novosibirsk, Russia
}

\begin{abstract}
Dynamic model of centrifugal pump with induction motor on the base of Suter curves is described. The model is implemented in surge model of PASS/Hydrosystem software. The ways to provide consistency between transient and steady state flow models are proposed. A rule of thumb is described to preliminarily estimate if pump start-up and shut down can produce significant pressure picks that require detailed simulation.
\end{abstract}

\section{Introduction}

Correct simulating of centrifugal pump dynamic behaviour during transient flow (and especially waterhammer) in pipeline transporting liquids is very important. Unfortunately there are many poorly investigated problems in this field.

This article presents dynamic model of centrifugal pumps with induced motor implemented in surge module of PASS/Hydrosystem software (more on the program and the model see in $[1,2,3])$.

The module currently supports four types of transient processes which require pump dynamic modeling. Two of them (pump start-up and shut down) deal with the pump as the origin of transient process. Two other cases are caused by changes in the other elements of piping system, but pump transient behavior is also important. These cases include the transient process with operating pump (when its duty point changes), and a rare case of transient flow through not operating, but hydraulically not isolated pump (with flow through it). Transient processes related to active automatic pump control, for example pumps with variable frequency drive, are scheduled to be implemented in the nearest future.

\section{Mathematical model of centrifugal pump with induced motor}

The behavior of a pump is controlled by the following torque balance equation $[4,5,6]$

$$
2 \pi J \frac{d n}{d t}=T_{\text {motor }}(n)-T_{\text {pump }}(Q, n)
$$

Where $n$ is pump rotation speed, rotations per sec, $t$ - time, sec; $Q$ - pump volume flow rate, $\mathrm{m}^{3} / \mathrm{sec} ; T_{\text {motor }}$ - motor torque, $\mathrm{N} \mathrm{m}$ (it equals zero for non-operating pump); $T_{\text {pump }}-$ torque from liquid on pump, $\mathrm{N} \mathrm{m}$; - total mass moment of inertia, $\mathrm{kg} \mathrm{m}^{2}$, which is the sum

${ }^{*}$ Leonid Korelstein: Korelstein@truboprovod.ru 
of moments of inertia of motor rotor, pump impeller and other rotating pump elements, and also the fluid in the pump

$$
J=J_{\text {rotor }}+J_{\text {impeller }}+J_{\text {fluid }}
$$

Note that $J_{\text {rotor }}$ is usually the largest term in (2), and its value, unlike the one of other terms, is almost always known from the manufacturer.

The boundary conditions at pump entrance and exit are defined by equation

$$
H_{\text {outlet }}-H_{\text {inlet }}=H(Q, n)
$$

where $H_{\text {inlet }}$ and $H_{\text {outlet }}$ - heads at pump entrance and exit, m; $H(Q, n)$ - pump head.

Combining equations (1) and (3) with standard waterhammer equations (written using Riemann invariants [3]) provides complete system of ordinary and partial differential equations. The resulting system is solved in PASS/Hydrosystem via finite difference method using explicit schema [3].

Thus, the problem is to obtain the functions $T_{\text {motor }}(n), T_{\text {pump }}(Q, n), H(Q, n)$.

It is known that the induced motor rotor rotation speed $n$ differs from the magnetic field rotation speed in stator $n_{s}$ (that can be calculated as $n_{s}=\frac{2 f}{p}$, where $f$ is the current frequency, Hz; $p$ - the number of stator poles). This difference is called slip and can be defined as $s=\frac{n_{s}-n}{n_{s}}$. Typical dependency of induce motor torque on slip is shown on Fig. 1.

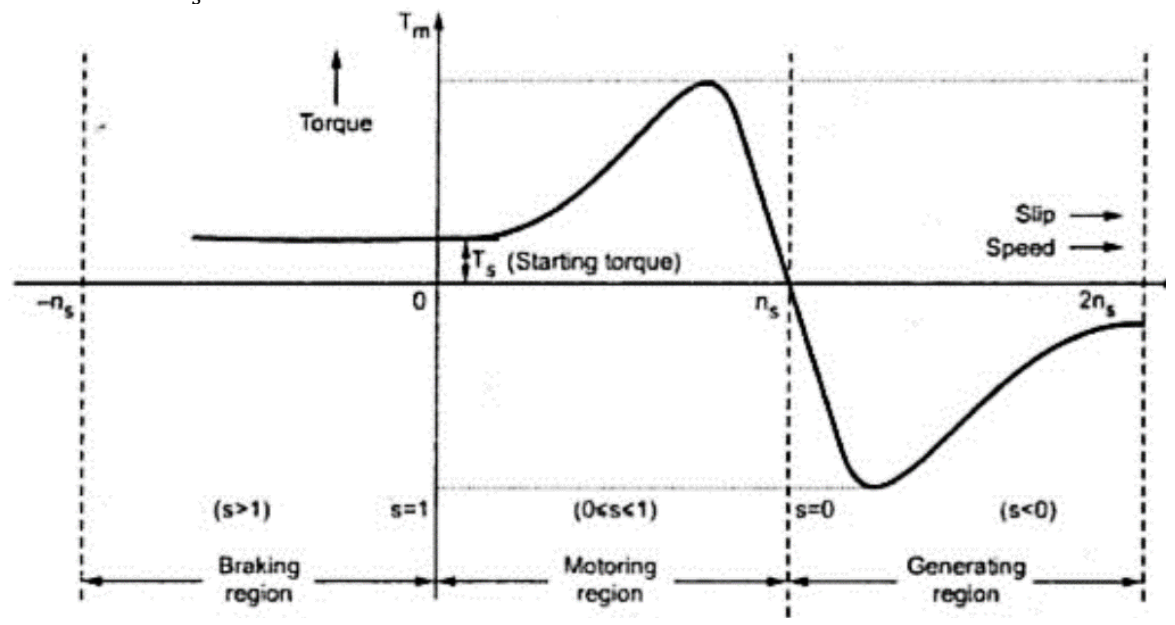

Fig. 1. Induced motor torque vs slip/rotation speed

In operating mode induced motor parameters are «floating» near nominal values $T_{\text {nom }}$ and $n_{\text {nom }}$, with slip being in the interval between zero and so called critical slip $s_{c r}$, which corresponds to maximum value of motor torque $T_{c r}$. The moment versus slip dependency for practical calculations is well described by Kloss equation [7]

$$
T_{\text {motor }}(n)=\frac{2 T_{c r}}{\frac{s}{s_{c r}}+\frac{s_{c r}}{s}}
$$

Note that critical and nominal motor parameters are practically always available from motor manufacturer.

The main problem is to calculate $T_{\text {pump }}(Q, n), H(Q, n)$, as during transient process the pump can be far outside its recommended by manufacturer operation zone and even out of the $1^{\text {st }}$ quadrant (i.e. positive values of $Q$ and $n$ ). We can have $Q$ and $n$ in other quadrants (four quadrant mode), with pump operating in energy dissipation, normal turbine, reverse turbine and reverse pumping modes $[4,5,6]$. Pump manufacturers usually don't provide any data on their pumps behavior in these modes. 
The first step to solve this problem is to reduce its dimension. If we simulate turbulent quadratic flow only (that covers most of practical cases) and take into account affinity laws, then $T_{\text {pump }}(Q, n), H(Q, n)$ can be presented in the following one-dimension way (proposed by Suter $[8,9])$ via dimensionless torque and head curves:

$$
\begin{gathered}
T_{\text {pump }}(Q, n) / T_{\text {nom }}=W_{T}^{2}(\theta)\left[\left(Q / Q_{\text {nom }}\right)^{2}+\left(n / n_{\text {nom }}\right)^{2}\right] \operatorname{sign}\left[W_{T}(\theta)\right] \\
H(Q, n) / H_{\text {nom }}=W_{H}^{2}(\theta)\left[\left(Q / Q_{\text {nom }}\right)^{2}+\left(n / n_{\text {nom }}\right)^{2}\right] \operatorname{sign}\left[W_{H}(\theta)\right]
\end{gathered}
$$

Where angle $\theta$ is defined as

$$
\theta=\tan ^{-1}\left(\frac{n}{n_{n o m}} \frac{Q_{n o m}}{Q}\right)
$$

All the nominal values correspond to pump BEP.

The following equations obtained from (5). (6)

$$
\begin{aligned}
& W_{T}(\theta)=\operatorname{sign}(T) \sqrt{\frac{|T| / T_{\text {nom }}}{\left(Q / Q_{\text {nom }}\right)^{2}+\left(n / n_{\text {nom }}\right)^{2}}} \\
& W_{H}(\theta)=\operatorname{sign}(H) \sqrt{\frac{|H| / H_{\text {nom }}}{\left(Q / Q_{\text {nom }}\right)^{2}+\left(n / n_{\text {nom }}\right)^{2}}}
\end{aligned}
$$

can be used to define functions $W_{T}(\theta)$ and $W_{H}(\theta)$ via experimental data obtained by testing specific pumps. Such curves then usually are extrapolated to similar pumps, with specific speed value $N_{S}=\frac{n_{n o m} Q_{n o m}}{H_{n o m}^{0.75}}$ [10] as measure of pump similarity. Most often the data for 3 specific speed values are used (see [11]). Correspondent curves are shown on Fig. 2. Additional data can be found in [11-18], and [19, 20] contain systematic critical review of all available experimental data.

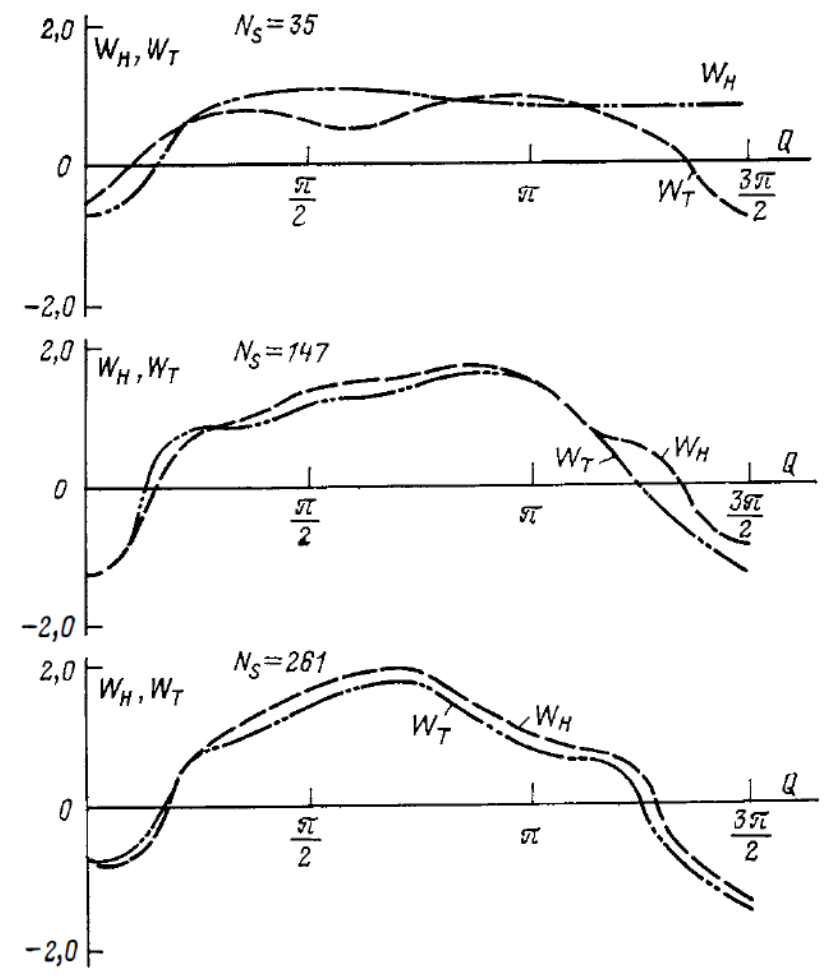

Fig. 2. Sutor curves for 3 values of specific speed

\section{Static and dynamic model consistency}


When implementing the model described above in software the authors were concerned by its consistency with the model used for steady state flow simulation. The transient process starts from steady state flow and should converge to steady state flow, and if these models are inconsistent, some parameters after simulation may appear to have vibrations that does not really exist. For steady-state flow the program uses traditional pump curves provided by the pump manufacturer for specific pumps, first of all head vs flow rate curve. To provide consistency it is necessary to partially correct curve $W_{H}(\theta)$ using (9) equation in the range of argument parameter values corresponding to the range of vendor traditional pump head vs flow rate curve. This idea is proposed for example in [21]. However, there is one nontrivial aspect - experimental data for traditional head-flow rate curve for pump with induced motor have been obtained for non-fixed value of pump rotation speed! This speed is nominal only in BEP, and its value is slightly "floating" around nominal values in the interval between $n_{s}$ and $n_{c r}$. These deviations are not so big, and usually are not taken into account nor in head vs flow rate curve, nor during steady state calculations using this curve. But they can seriously affect the results of dynamic simulation!

In order to take these deviations into account, the procedure of partial correction of Suter head curve should be the following. First use the following steady state variant of equation (1)

$$
T_{\text {motor }}(n)=W_{T}^{2}(\theta)\left[\left(Q / Q_{\text {nom }}\right)^{2}+\left(n / n_{\text {nom }}\right)^{2}\right] T_{\text {nom }}
$$

along with equations (4) and (7) for set value of $Q$ or $\theta$, and find correspondent value of $n$ from these equations, and only then use (9) substituting this value to correct $W_{H}(\theta)$.

Another consistency problem is using the correct value of resistance coefficient for minor losses on pump in case when it is switched off but not hydraulically isolated. This value is different for direct and reverse flow through the pump and can be estimated in the following way. First zeros of function $W_{T}(\theta)$ should be found, with zero $\theta_{d}$ in the 1st quadrant corresponds to direct flow and zero $\theta_{r}$ in the $3^{\text {rd }}$ quadrant - to reverse flow. Then resistant coefficient for direct and reverse flow (reduced to velocity in pump inlet nozzle with inner diameter $D$ ) can be calculated by the following formula (substituting $\theta_{d}$ and $\theta_{r}$ )

$$
\zeta=0.125 g \pi^{2} D^{4} W_{H}^{2}(\theta) H_{\text {nom }} Q_{n o m}^{-2}\left[1+\tan ^{2}(\theta)\right]
$$

\section{Rule of thumb when pump start-up/shut down waterhammer is significant}

Main equation (1) can be written in the following dimensionless form

$$
T_{r} \frac{d \beta}{d t}=\frac{1}{T_{\text {nom }}}\left[T_{\text {motor }}(\beta)-T_{\text {pump }}(q, \beta)\right]
$$

Where $\beta=n / n_{\text {nom }}, q=Q / Q_{\text {nom }}$, and the only dimension parameter

$$
T_{r}=2 \pi J n_{\text {nom }} / T_{\text {nom }}>2 \pi J_{\text {motor }} n_{\text {nom }} / T_{\text {nom }}
$$

is time characterizing pump transient processes (usually from 0.05 till $5 \mathrm{sec}$ ).

Software simulation shows that the following rule of thumb can be proposed - if $T_{r}$ is much longer than time waterhammer waves need to travel from pump to piping end nodes and back, then pump start-up and shut down don't produce significant pressure picks. Otherwise pressure variations can be significant and detailed simulation should be recommended.

Let's illustrate this rule by results of simulating simple pipeline with the pump in the beginning of the pipe and relatively long $(600 \mathrm{~m})$ outlet pipe. The result of shut down simulation is shown on Fig. 3 and Fig. 34 for $T_{r}$ values 0.2 and 2 sec. Points 1, 2 and 3 correspond to pump inlet nozzle, outlet nozzle and the middle of outlet pipe. 
Fig. 3. Pump shut down, $T_{r}=0.2 \mathrm{sec}$

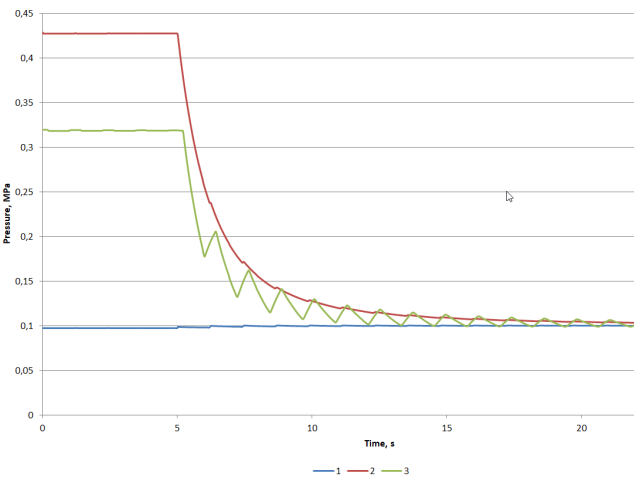

Fig. 4. Pump shut down, $T_{r}=2 \mathrm{sec}$

The results for pump start-up are shown on Fig. 5 and Fig. 56.

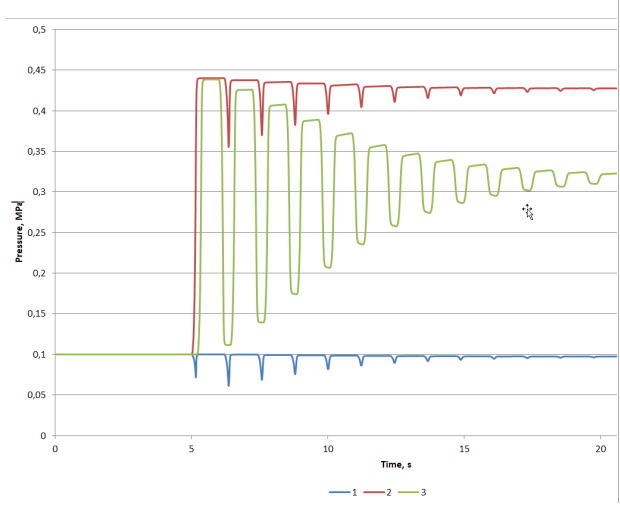

Fig. 5. Pump start-up, $T_{r}=0.2 \mathrm{sec}$

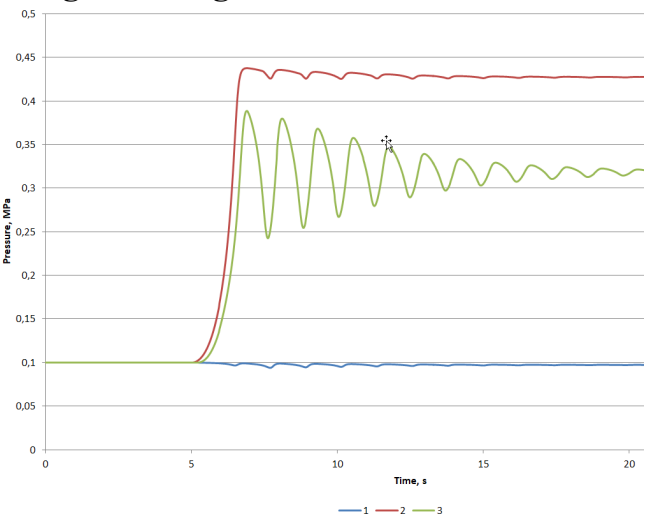

Fig. 6. Pump start-up, $T_{r}=2 \mathrm{sec}$

\section{Problems for further investigation}

There are many aspects of pump dynamic simulation not covered in this article which are waiting for detailed research - such as high viscosity liquid transient flow in pumps, settling slurries transient flow, cavitation influence on pump dynamic behavior etc. We hope to address some of them in future articles.

\section{References}

1. E. Yudovina, E. Pashenkova, L. Korelstein, In: Piping systems of Energetics: Methodical and applied problems of mathematical simulation, 438 (Novosibirsk, Nauka, 2015) [in Russian]

2. A. Babenko, E. Yudovina, L. Korelstein, T. Gartman, Program products and systems, 1, 141 (2013) [in Russian]

3. E. Yudovina, S. Lisin, V. Tarasevich, A. Lee, A. Moroz, In: Piping systems of Energetics: Mathematical and computer technologies of intellectualization, 353 (Novosibirsk, Nauka, 2017) [in Russian]

4. J.A. Fox, Hydraulic Analysis of Unsteady Flow in Pipe Networks (London, 1977) 
5. E.B. Wylie, V.L. Streeter, Fluid transients (McGraw-Hill International Book Company, New York, USA, 1978)

6. M.H. Chaudhry, Applied Hydraulic Transients. 3rd Edition (Springer, 2014)

7. K. Hameyer. Electrical Machines 1. Basics, Design, Function, Operation (IEM RWTH, 2001)

8. M. Marchal, G. Flesch, P. Suter, In: Proceedings of the Int. Symp. on Waterhammer in Pumped Storage Projects, ASME, Chicago, USA, 67 (1965)

9. Suter, P., Sulzer Technical Review Research, 1966, 45 (2010)

10. ANSI-HI 1-1-1.2-2014. Rotodynamic Centrifugal Pumps for Nomenclature and Definitions (2014)

11. B. Donsky, J. Basic Engineering, December, 685 (1961)

12. R.T. Knapp, Trans.,Amer. Soc. of Mech. Engrs., 59, 683 (1937)

13. W.M. Swanson, Trans. Amer. Soc. of Mech. Engrs., 75, 819 (1953)

14. C.P. Kittredge, ASME Journal of Basic Engineering, 78, 6, 1307 (1956)

15. R.J. Brown, D.C. Rogers, ASME Journal of Mechanical Design, 102, 4, 807 (1980)

16. A.R.D. Thorley, A. Chaudry, In: Proc. 7th Int. Conf. On Pressure Surges, BHRA, Cranfield, Beds, UK, 461 (1996)

17. E. Ayder, C. Ozgur, A.N. Ilikan, L. Kavurmacioglu, M. Sen, K. Kirkkopru, In: Proc. of the ASME Fluids Engineering Division Summer Meeting (FEDSM2009), Vail USA, August 2-6 (2009)

18. Z. Giljen, M. Nedeljković, Y. Cheng, 28th IAHR Symposium on Hydraulic Machinery and Systems (2016)

19. , C.S. Martin, ASME/FED, 6, 1 (1983)

20. W. Trey, D. Trygve, D.C. Rogers, In: Proc. the ASME 2020 Pressure Vessels and Piping Conference PVP2020 July 19-24 (Minneapolis, MN, USA, 2020)

21. W. Wan, W. Huang, J. Mech. Science and Technology, 25(10), 2583 (2011) 